\title{
Orienting the superficial inferior epigastric artery (SIEA) pedicle in a stacked SIEA-deep inferior epigastric perforator free flap configuration for unilateral tertiary breast reconstruction
}

\author{
Ya-han $\mathrm{Yu}^{1,2}$, Dina Ghorra ${ }^{3,4}$, Christine Bojanic ${ }^{4}$, Oti N. Aria ${ }^{4,5}$, Louise MacLennan ${ }^{4}$, \\ Charles M. Malata ${ }^{4,6,7}$ \\ ${ }^{1}$ Clinical School of Medicine, University of Cambridge, Cambridge, UK; ${ }^{2}$ School of Medicine, National Taiwan University, Taipei, Taiwan; \\ ${ }^{3}$ Department of Plastic and Reconstructive Surgery, Alexandria Faculty of Medicine, Alexandria University Hospital, Alexandria, Egypt; \\ ${ }^{4}$ Department of Plastic and Reconstructive Surgery, Addenbrooke's University Hospital, Cambridge University Hospitals NHS Foundation \\ Trust, Cambridge, UK; ${ }^{5}$ Plastic and Reconstructive Surgery Unit, Surgery Department, University of Port Harcourt Teaching Hospital, Port \\ Harcourt, Nigeria; ${ }^{6}$ Cambridge Breast Unit, Addenbrooke's University Hospital, Cambridge University Hospitals NHS Foundation Trust, \\ Cambridge; ${ }^{7}$ Anglia Ruskin University School of Medicine, Chelmsford, UK
}

Superficial inferior epigastric artery (SIEA) flaps represent a useful option in autologous breast reconstruction. However, the short-fixed pedicle can limit flap inset options. We present a challenging flap inset successfully addressed by de-epithelialization, turnover, and counterintuitive rotation. A 47-year-old woman underwent left tertiary breast reconstruction with stacked free flaps using right deep inferior epigastric perforator and left SIEA vessels. Antegrade and retrograde anastomoses to the internal mammary (IM) vessels were preferred; additionally, the thoracodorsal vessels were unavailable due to previous latissimus dorsi breast reconstruction. Optimal shaping required repositioning of the lateral ends of the flaps superiorly, which would position the ipsilateral SIEA hemi-flap pedicle lateral to and out of reach of the IM vessels. This problem was overcome by turning the SIEA flap on its long axis, allowing the pedicle to sit medially with the lateral end of the flap positioned superiorly. The de-epithelialized SIEA flap dermis was in direct contact with the chest wall, enabling its fixation. This method of flap inset provides a valuable solution for medializing the SIEA pedicle while maintaining an aesthetically satisfactory orientation. This technique could be used in ipsilateral SIEA flap breast reconstructions that do not require a skin paddle, as with stacked flaps or following nipple-sparing mastectomy.

Keywords Tertiary breast reconstruction / Microsurgical free flap / SIEA-DIEP flap / Bipedicled free flap
Correspondence: Charles M. Malata Department of Plastic and Reconstructive Surgery, Addenbrooke's University Hospital, Cambridge University Hospitals NHS Foundation Trust, Cambridge, CB2 200, UK Tel: +44-1223-586672 Fax: +44-1223-257177 E-mail:cmalata@hotmail.com

Received: September 21, 2019 • Revised: December 11, $2019 \bullet$ Accepted: December 29, 2019

pISSN: 2234-6163 • elSSN: 2234-6171 • https://doi.org/10.5999/aps.2019.01319 • Arch Plast Surg 2020;47:473-477 


\section{INTRODUCTION}

The use of stacked bipedicled abdominal flaps for unilateral breast reconstruction has been extensively described. These flaps include traditional pedicled transverse rectus abdominis myocutaneous (TRAM) [1], free TRAM [2], free deep inferior epigastric perforator (DIEP) [3], and free superficial inferior epigastric artery (SIEA) flaps [4-6]. Although combined SIEA-DIEP bipedicled folded and coned free flaps have been described [4-7], there is a lack of information in the literature on the simultaneous use of stacked flaps of different types from each hemi-abdomen for unilateral reconstruction. Only one report has described a stacked combination of a SIEA and a DIEP flap [8].

The advantages of stacked flaps include increased available flap volume and flap projection as well as the enabling of favorable dimensions for the reconstructed breast. A critical step in the successful execution of double-pedicled flaps is the design of the flap orientation and inset $[4,7,9]$. Possible flap insets include folded, coned, stacked, and horizontal or vertical adjacent flap variations $[4,7,9]$. The anastomotic configuration of stacked flaps can be either one flap on top of another buried flap with intra-flap anastomoses, or one flap on top of the other with both flaps vascularized with independent extra-flap anastomoses. Although numerous flap orientations exist, these are limited by the length and positioning of the flap pedicle. This is less pertinent when SIEA flaps are used than when DIEP flaps are utilized.

We present a method of inset and orientation that uses a turned over, completely de-epithelialized, buried SIEA flap in a stacked SIEA-DIEP free flap configuration in a patient undergoing unilateral salvage breast reconstruction. This method allowed for the creation of a successful SIEA vascular pedicle anastomosis to the ipsilateral internal mammary (IM) vessels, while maintaining the preferred orientation for an aesthetically optimal outcome.

\section{CASE}

A 47-year-old woman presented for revision of a reconstruction procedure in her left breast after undergoing a left skin-sparing mastectomy and immediate reconstruction with a latissimus dorsi myocutaneous flap and expandable implant 6 years prior. Her treatment subsequent to the mastectomy included chemotherapy, external beam radiotherapy, and 3 years later, a capsulectomy and implant exchange for a fixed-volume implant. She complained of considerable discomfort in the reconstructed breast as well as suboptimal shape (Fig. 1A and B), in particular an asymmetrical breast base compared to the contralateral breast. The patient also complained of capsular contracture, and she was keen to avoid further revisions or capsulectomies in the future. She was otherwise a fit and healthy non-smoker with a body mass index of $26.5 \mathrm{~kg} / \mathrm{m}^{2}$ and a $36 \mathrm{C}$ bra size.

In view of the patient's limited abdominal tissue and the broad dimensions of her contralateral breast (Fig. 1A and B), the use of a bipedicled stacked DIEP flap anastomosed anterogradely in the second intercostal space and retrogradely in the third intercostal space was planned. Preoperative computed tomography (CT) angiography confirmed that this was feasible, although the left side had only a single usable perforator with a long (6 $\mathrm{cm}$ ) intramuscular course.

Intraoperatively, a left partial capsulectomy with removal of a 532-g implant was performed via a peri-areolar incision that included the latissimus dorsi skin paddle. A 19-cm right hemiDIEP flap pedicle weighing $527 \mathrm{~g}$ was harvested with two lateral

\section{Fig. 1. Preoperative and postoperative images}

A 47-year-old woman underwent unilateral left salvage breast reconstruction with stacked abdominal free flaps utilizing right deep inferior epigastric perforator and left free superficial inferior epigastric artery vessels. (A, B) Preoperative images demonstrate a suboptimal cosmetic appearance, including significant asymmetry, rippling, synmastia, and a laterally displaced latissimus dorsi flap skin paddle. (C, D) Postoperatively, the patient had a more naturallooking salvaged left breast with improved symmetry, no rippling, and a better-positioned skin paddle. She subsequently declined contralateral mastopexy.
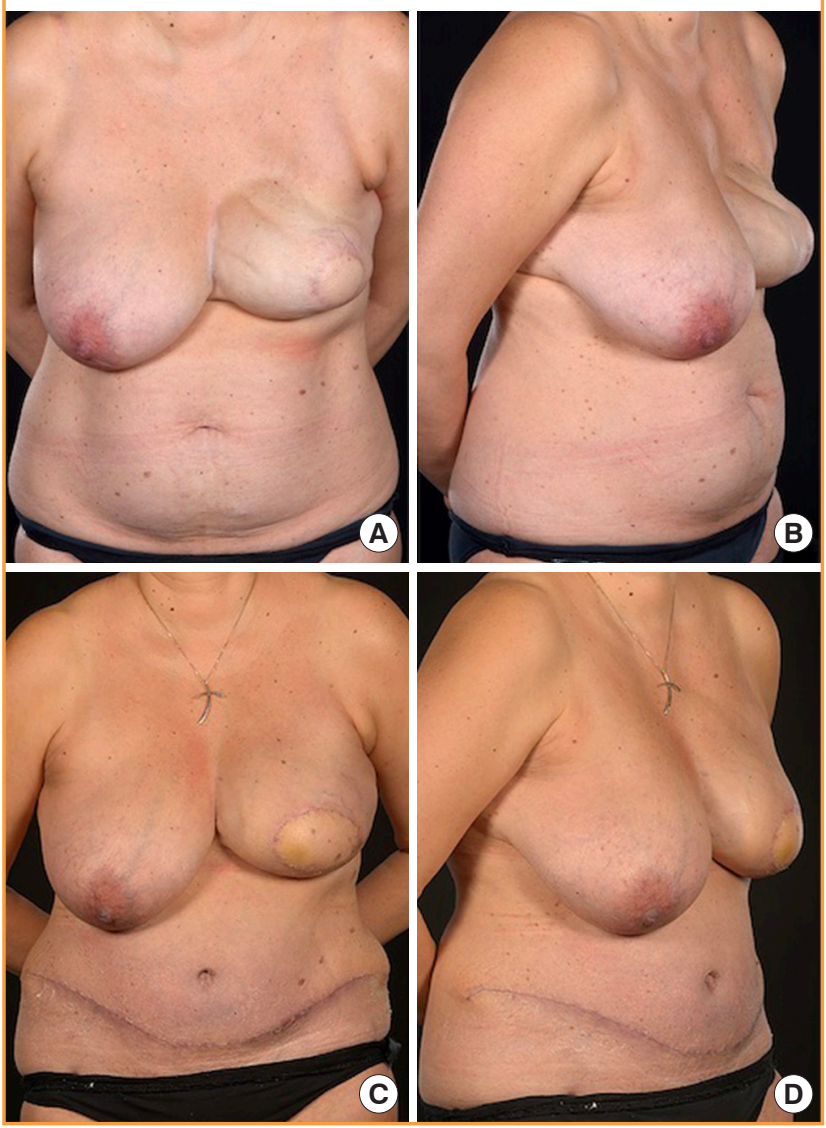
row perforators. Dissection of the left hemi-abdomen revealed a large SIEA and a large superficial inferior epigastric vein. In view of the CT angiography findings of a single DIEP perforator with unfavorable anatomy, the left hemi-flap was raised as a $7-\mathrm{cm}$ long SIEA flap pedicle with a weight of $463 \mathrm{~g}$. A standard flap harvest following the vascular pedicle to its origin from the femoral artery and vein was performed. It was noted that the vessel caliber increased substantially as the dissection progressed towards the femoral vessels (Fig. 2A). The left IM vessels were exposed in the second and third intercostal spaces using our standard total rib preservation technique [10]. Using the thoracodorsal vascular pedicle for anastomosis was not possible, as it had been previously used for the latissimus dorsi flap reconstruction. For aesthetic reasons, the lateral parts of the two hemi-flaps were positioned superiorly to facilitate a smooth takeoff while maximizing the projection of the lower part of the breast.

The DIEP flap from the right hemi-abdomen was rotated $90^{\circ}$ clockwise (Fig. 2B), positioned superficially, and anastomosed in an antegrade manner to the IM vessels in the second intercostal space. The SIEA flap from the ipsilateral left hemi-abdomen was planned to be buried deep to the DIEP flap in a stacked fashion. However, it was noted that to rotate the SIEA flap so that the lateral part of the flap would superior and in the aesthetically ideal position would have resulted in the short, fixed vascular pedicle being positioned laterally out of the range of the exposed recipient IM vessels. This would also have resulted in the unavailability of any thoracodorsal recipient. This issue was resolved by rotating the SIEA flap $90^{\circ}$ anticlockwise and then turning the flap over on its long axis (now vertical) to allow the pedicle to be positioned medially (Fig. 2C). The SIEA pedicle was then anastomosed in a retrograde fashion to the IM vessels in the third intercostal space. Both flaps demonstrated good immediate flow.

The flaps were maintained in their stacked position with the DIEP flap placed superficially and the SIEA flap deep to the DIEP flap (Fig. 2D). The SIEA flap dermis, which was now in direct apposition with the chest wall, was completely de-epithelialized and sutured to the deep fascia of the chest wall using 2-0 polydioxanone sutures. The skin of the DIEP flap, positioned superficially, was de-epithelialized except for a central skin paddle for flap monitoring (Fig. 1C). The two flaps were sutured together with 3-0 Monocryl sutures. There were no perioperative or postoperative complications, and the patient was fit for

\section{Fig. 2. Schematic diagram}

Orienting the hemi-abdominal free flaps during the surgery. (A) A right deep inferior epigastric perforator (DIEP) flap and a left free superficial inferior epigastric artery (SIEA) flap were harvested. (B) The DIEP flap from the right hemi-abdomen was rotated $90^{\circ}$ clockwise, positioned superficially, and anastomosed antegrade to the internal mammary vessels in the second intercostal space. (B) The SIEA flap was rotated $90^{\circ}$ anticlockwise. (C) The SIEA flap was then turned over on its long axis to allow the pedicle to be positioned medially and anastomosed retrograde to the internal mammary vessels in the third intercostal space. (D) The SIEA flap dermis was now in direct apposition with the chest wall and fixed to the pectoral fascia.
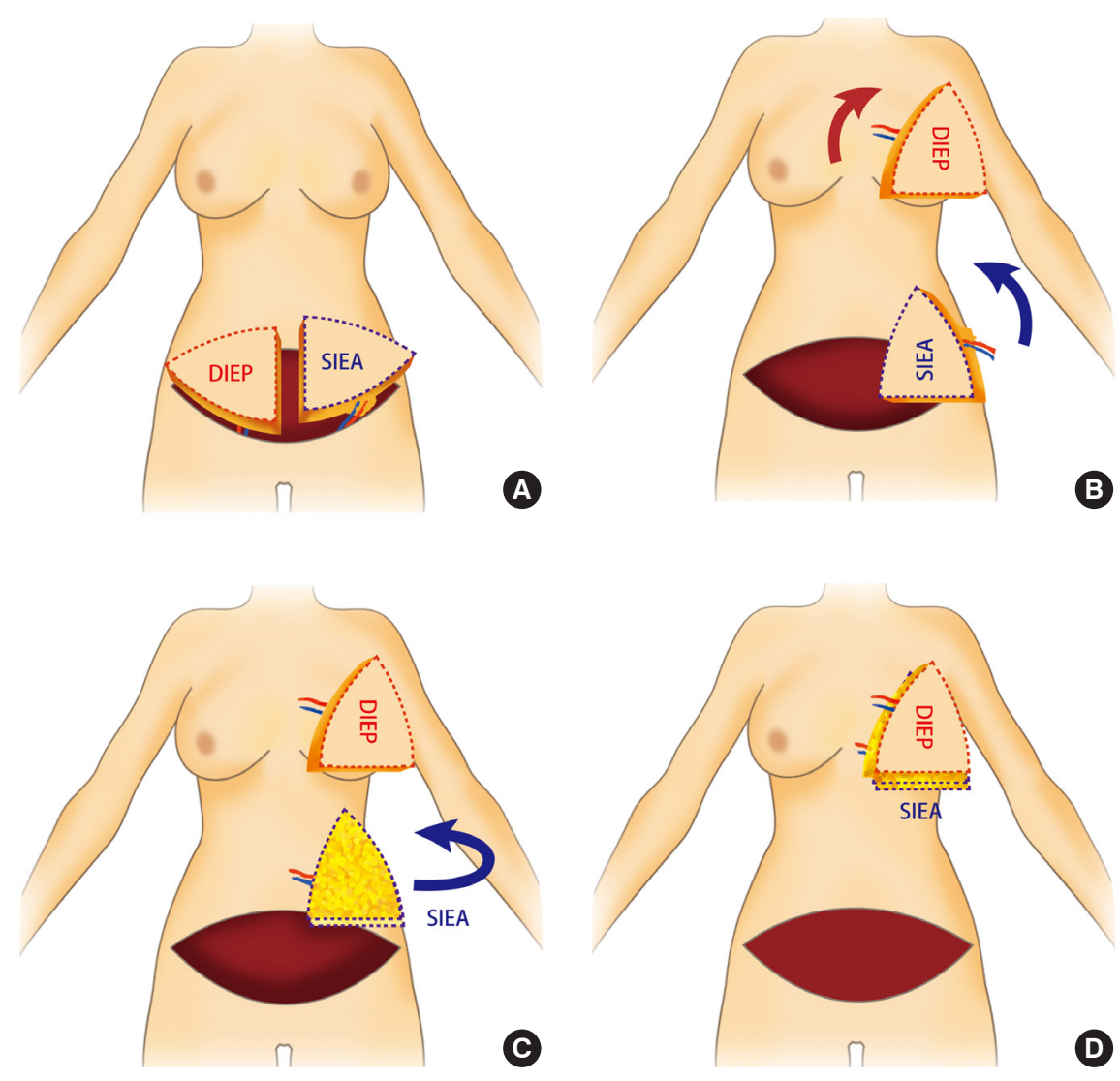
discharge on day 7, with the actual discharge occurring 3 days later for social reasons.

\section{DISCUSSION}

Although the SIEA flap is considered the ideal lower abdominal flap due to its low donor site morbidity [11], it has a number of disadvantages. Notable among these is its short vascular pedicle and variable arterial anatomy [12]. In addition, a key problem with SIEA flaps pertinent to flap inset is the "tadpole" configuration of the pedicle; that is, the pedicle enters the flap at the edge [13], in contrast to the "mushroom" configuration of the DIEP flap and its vascular pedicle. This relative fixity in the edge of the flap from which the pedicle arises, along with the short nature of the pedicle, limits the flexibility with which the flap can be oriented, as the most inferior part of the SIEA flap will inevitably need to be rotated into a medial position to facilitate anastomosis to the IM vessels. For this reason, SIEA flaps that will be anastomosed to the IM vessels should ideally be harvested from the contralateral side. This is because rotation of the inferior portion of the flap medially results in the relatively thin lateral part of the flap being positioned superiorly, ensuring a smooth take-off, while the relatively fuller medial part of the flap is positioned inferiorly, optimizing lower pole projection. In our case, the left ipsilateral hemi-flap was found intraoperatively to be ideal for harvest as an SIEA, as supported by the CT angiogram findings. The ipsilateral SIEA flap was rotated $90^{\circ}$ anticlockwise into the left breast (as required to provide ideal flap orientation), causing the pedicle to point laterally and therefore be out of reach of the IM vessels. Therefore, we turned the flap over along its long axis to place the dermal surface against the chest wall. This direct apposition of the dermis and chest wall had the additional benefit of facilitating suturing of the SIEA flap dermis to the chest wall fascia while avoiding compression of the flap fat around the flap periphery.

Using medial rather than lateral recipient vessels is particularly helpful in optimizing the breast shape [4] for short-pedicled SIEA flaps, as it allows for more medial placement of the flap and thus better cleavage (Fig. 1C). Utilizing vessels in the axilla as recipients for the SIEA flap could result in overly lateral placement of the breast mound in order to overcome the short length of the pedicle.

A drawback of the inset technique we have described is the difficulty of postoperative monitoring of the buried SIEA flap. In the absence of a skin paddle on the surface, the completely buried SIEA flap was monitored with Doppler ultrasound of its pedicle at the third intercostal space. In our experience, retrograde flow from the IM vessels has a different quality on Dop- pler ultrasound from anterograde flow, which can be utilized to differentiate between pedicles in cases involving a bipedicled free flap in which one hemi-flap is anastomosed anterograde and the other hemi-flap is anastomosed retrograde to the IM vessels. Alternatively, an implantable Swartz device for venous Doppler ultrasonography may be considered for postoperative monitoring of buried flaps, and the choice of a monitoring method should be based on the surgeon's preference and experience, as well as equipment availability.

The technique reported herein proved useful for overcoming difficulties related to the pedicle position for an ipsilateral SIEA flap, enabling anastomoses to the IM vessels. By turning the flap over, a completely buried SIEA flap pedicle can be medialized and hence easily anastomosed to the IM vessels without sacrificing the aesthetically optimal orientation. This technique resulted in an excellent shape and should be considered when faced with a similar problem in the use of stacked abdominal free flaps incorporating an ipsilateral SIEA pedicle.

\section{NOTES}

\section{Conflict of interest}

No potential conflict of interest relevant to this article was reported.

\section{Ethical approval}

The study was performed in accordance with the principles of the Declaration of Helsinki. Written informed consent was obtained.

\section{Patient consent}

The patient provided written informed consent for the publication and the use of her images.

\section{Author contribution}

Conceptualization: CM Malata. Visualization: Y Yu. Writing original draft: Y Yu. Writing - review \& editing: all authors. Project administration: CM Malata, C Bojanic. Approval of final manuscript: all authors.

\section{ORCID}

Ya-han Yu https://orcid.org/0000-0003-3695-9494

Dina Ghorra https://orcid.org/0000-0001-7659-9863

Christine Bojanic

Oti N. Aria https://orcid.org/0000-0003-1708-3909

Charles M. Malata https://orcid.org/0000-0002-0070-0838 


\section{REFERENCES}

1. Spear SL, Travaglino-Parda RL, Stefan MM. The stacked transverse rectus abdominis musculocutaneous flap revisited in breast reconstruction. Ann Plast Surg 1994;32:565-71.

2. Arnez ZM, Scamp T. The bipedicled free TRAM flap. Br J Plast Surg 1992;45:214-8.

3. DellaCroce FJ, Sullivan SK, Trahan C. Stacked deep inferior epigastric perforator flap breast reconstruction: a review of 110 flaps in 55 cases over 3 years. Plast Reconstr Surg 2011; 127:1093-9.

4. Malata CM, Rabey NG. Decision making in double-pedicled DIEP and SIEA abdominal free flap breast reconstructions: an algorithmic approach and comprehensive classification. Front Surg 2015;2:49.

5. Hamdi M, Khuthaila DK, van Landuyt K, et al. Doublepedicle abdominal perforator free flaps for unilateral breast reconstruction: new horizons in microsurgical tissue transfer to the breast. J Plast Reconstr Aesthet Surg 2007;60:90414.

6. Francis EC, Chan JY, Hussey AJ. A novel stacked SIEA flap for unilateral immediate breast reconstruction. J Reconstr Microsurg Open 2017;2:e49-51.

7. Patel NG, Rozen WM, Chow WT, et al. Stacked and bipedi- cled abdominal free flaps for breast reconstruction: considerations for shaping. Gland Surg 2016;5:115-21.

8. Figus A, Fioramonti P, Ramakrishnan V. Stacked free SIEA/ DIEP flap for unilateral breast reconstruction in a thin patient with an abdominal vertical midline scar. J Reconstr Microsurg 2007;23:523-5.

9. Chang EI, Kronowitz SJ. Dual-pedicle flap for unilateral autologous breast reconstruction revisited: evolution and optimization of flap design over 15 years. Plast Reconstr Surg 2016;137:1372-80.

10. Malata CM, Moses M, Mickute Z, et al. Tips for successful microvascular abdominal flap breast reconstruction utilizing the "total rib preservation" technique for internal mammary vessel exposure. Ann Plast Surg 2011;66:36-42.

11. Arnez ZM, Khan U, Pogorelec D, et al. Breast reconstruction using the free superficial inferior epigastric artery (SIEA) flap. Br J Plast Surg 1999;52:276-9.

12. Cormack GC, Lamberty BG. The arterial anatomy of skin flaps. Edinburgh: Churchill Livingstone; 1994.

13. Miyagi K, Di Candia M, Patel AJK, et al. Avoiding pitfalls in microvascular breast reconstruction. In: Shiffman MA, editor. Breast reconstruction. New York: Springer; 2016. p. 57192. 